\title{
MELHORIA DA QUALIDADE DE UVA 'BORDÔ' PARA PRODUÇÃO DE VINHO E SUCO DE UVA ${ }^{1}$
}

\author{
FRANCELIZE CHIAROTTI ${ }^{2}$, INÊS THOMAZ GUERIOS ${ }^{3}$, FRANCINE LORENA CUQUEL ${ }^{4}$, \\ LUIZ ANTONIO BIASI ${ }^{5}$
}

RESUMO - Visando à produção de vinho e suco de uva de melhor qualidade, o objetivo desta pesquisa foi aumentar a relação de sólidos solúveis totais/acidez total titulável (SST/ATT), conhecida como flavor, da uva 'Bordô', com o uso de reguladores vegetais e avaliar os efeitos destes produtos nas demais características físico-químicas dos frutos. O trabalho foi realizado durante duas safras, 2009/2010 e 2010/2011, em Bocaiuva do Sul-PR. As variáveis físicas analisadas foram: comprimento e largura dos cachos; diâmetro das bagas; número de bagas por cacho; massa dos cachos e bagas; e número de sementes por baga. As variávies químicas foram: sólidos solúveis totais (SST); acidez total titulável (ATT); pH e SST/ATT. A aplicação de $\mathrm{GA}_{3}$ a $100 \mathrm{mg} \mathrm{L}^{-1}$ propiciou melhoria da qualidade dos frutos para a produção de vinho e suco de uva, além de maior desenvolvimento dos frutos.

Termos para indexação: ácido giberélico, thidiazuron, 6-benzilaminopurina, regulador de crescimento, viticultura, Vitis labrusca.

\section{IMPROVING THE QUALITY OF GRAPE 'BORDO’ FOR PRODUCTION OF WINE AND GRAPE JUICE}

\begin{abstract}
Aiming the production of wine and grape juice of better quality, the objective of this study was to increase the ratio of total soluble solids / titratable acidity (TSS / TA), known as flavor, of the grape 'Bordo' by the use of plant growth regulators and to evaluate the effects of these products on the other chemical and physical characteristics of the fruit. The work was conducted during two crop seasons, 2009/2010 and 2010/2011 in Bocaiuva do Sul, PR, Brazil. The physical variables analyzed were: length and width of clusters, berries diameter, number of berries per cluster, cluster and berries weight, and number of seeds per berry. The chemical variables were: soluble solids (TSS), titratable acidity (TTA), pH and TSS / TTA. The application of $\mathrm{GA}_{3}$ at $100 \mathrm{mg} \mathrm{L}^{-1}$ provided the improvement of fruit quality for wine and grape juice production, as well as the improvement of fruits development.
\end{abstract}

Index Terms: gibberellic acid, thidiazuron, 6-benzylaminopurine, plant growth regulator, viticulture,Vitis labrusca.

\section{INTRODUÇÃO}

As cultivares de Vitis labrusca e seus híbridos constituem a base da produção de vinhos de mesa e de suco de uva no Brasil e representam mais de $85 \%$ do volume de uvas industrializadas no País (CAMARGO et al., 2005). Entre as cultivares de Vitis labrusca, a 'Bordô' é bastante plantada no Brasil (32.000 t) para produção de vinho tinto, suco, vinagre, geleias e, por sua precocidade, é consumida in natura (RIZZON et al., 2000).

A elevada acidez é uma característica das prin- cipais cultivares de V. labrusca (RIZZON; MIELE, 2006). Isto leva a uma dificuldade para o plantio da uva 'Bordô' para produção de vinho e suco, uma vez que a relação obtida entre o teor de sólidos solúveis totais e a acidez total titulável (SST/ATT), conhecida como flavor, representa o equilíbrio entre o gosto doce e ácido do suco, portanto um indicativo de qualidade deste produto (RIZZON; LINK, 2006).

As caraterísticas ideais da uva para produção de vinhos tintos de qualidade são baixos teores de acidez, de 3,1 a 3,3 (RIZZON, 2004), teor de açúcares deve estar no mínimo com $14^{\circ}$ Brix e a relação

\footnotetext{
${ }^{1}$ Trabalho Sinfruit 144 - Simpósio Internacional de Fruticultura - Avanços na Fruticultura (17 a 21 Outubro)

${ }^{2}$ Eng ${ }^{\mathrm{a}}$.Agrônoma, Mestranda do Programa de Pós Graduação em Agronomia - Produção Vegetal- UFPR. (Bolsista REUNI). Rua dos Funcionários 1540, CEP.80035-050, Juvevê, Cutitiba-PR. E-mail: chiarotti.f@gmail.com

${ }^{3}$ Eng ${ }^{a}$.Agrônoma, Mestranda do Programa de Pós Graduação em Agronomia - Produção Vegetal- UFPR. E-mail: inestgg@yahoo.com.br

${ }^{4}$ Eng $^{\text {a }}$.Agrônoma, Dra. Professora Associada do Departamento de Fitotecnia e Fitossanitarismo daUFPR, E-mail: francine@ufpr.br

${ }^{5}$ Eng $^{\circ}$.Agronomo, Dr. Professor Associado do Departamento de Fitotecnia e Fitossanitarismo da UFPR. E-mail: biasi@ufpr.br
} 
entre o teor de sólidos solúveis totais e a acidez total titulável, entre 15 e 45 (BRASIL, 2004). Entretanto, Chouhury et al. (2001) consideram que oflavor desejável seja igual ou maior que 20 .

Via de regra, nas regiões Sul e Sudeste brasileiras, o período de maturação da cultivar Bordô coincide com o período chuvoso (REGINA et al., 2010), e há relatos dos produtores locais que, por esta razão, a uva 'Bordô' não atinge na colheita uma relação SST/ATT alta o suficiente para produzir bons vinhos. Isto aparentemente desqualifica o plantio de uva 'Bordô' nesta região para a atividade vinícola.

Para Tecchio et al. (2006), o uso de reguladores vegetais na viticultura melhora as características morfológicas dos cachos e bagas, entretanto há divergências sobre o efeito da aplicação de reguladores vegetais em uva sobre o teor de SST, tendo sido observado aumento (VIEIRA et al., 2008; CASANOVA et al., 2009) e diminuição deste teor (BYUN; KIM, 1995; BOTELHO et al., 2004; NACHTIGAL et al., 2005). Entretanto, estes autores não apresentaram os efeitos sobre a relação SST/ ATT, que representa um indicativo de qualidade para o vinho. O objetivo desta pesquisa foi aumentar a relação SST/ ATT da uva 'Bordô' com o uso de reguladores vegetais e avaliar os efeitos destes produtos nas demais características físico-químicas dos frutos.

\section{MATERIAL E MÉTODOS}

O experimento foi conduzido em um pomar de videira cv. Bordô, conduzido em espaldeira, com manejo convencional, no município de Bocaiuva do Sul-PR $\left(25^{\circ} 09^{\prime} 02.06^{\prime \prime} \mathrm{S}, 4^{\circ} 07^{\prime} 14.38^{\prime \prime}\right.$ O e $1052 \mathrm{~m}$ de altitude). As avaliações foram realizadas nas safras de 2009/2010 e 2010/2011. Os reguladores vegetais aplicados foram ácido giberélico $\left(\mathrm{GA}_{3}\right)$, produto comercial Pro-Gibb ${ }^{\circledR}\left(10 \% \mathrm{GA}_{3}\right)$, thidiazuron (TDZ) e 6-benzilaminopurina (BAP). A aplicação dos reguladores foi feita por imersão dos cachos nas referidas soluções, 14 dias após o pleno florescimento, adicionadas de adjuvante Tween $20^{\circledR}$ a $1 \%$. As concentrações utilizadas foram TDZ $5 \mathrm{mg}$ $\mathrm{L}^{-1}$, TDZ $10 \mathrm{mg} \mathrm{L}^{-1}$, TDZ $5 \mathrm{mg} \mathrm{L} \mathrm{m}^{-1}+\mathrm{GA}_{3} 100 \mathrm{mg}$ $\mathrm{L}^{-1}$, TDZ $10 \mathrm{mg} \mathrm{L}^{-1}+\mathrm{GA}_{3} 100 \mathrm{mg} \mathrm{L}^{-1}, \mathrm{GA}_{3} 100 \mathrm{mg}$ $\mathrm{L}^{-1}$, BAP $5 \mathrm{mg} \mathrm{L}^{-1}$, BAP $10 \mathrm{mg} \mathrm{L}^{-1}$, BAP $5 \mathrm{mg} \mathrm{L}^{-1}+$ $\mathrm{GA}_{3} 100 \mathrm{mg} \mathrm{L}^{-1}$, BAP $10 \mathrm{mg} \mathrm{L}^{-1}+\mathrm{GA}_{3} 100 \mathrm{mg} \mathrm{L}^{-1}$. Adicionalmente, foi efetuado um tratamento-controle sem a aplicação de reguladores vegetais. Foi utilizado delineamento experimental inteiramente casualizado, com 6 repetições e 2 cachos de uva por parcela, nas safras de 2009/2010 e 2010/2011. Quando os cachos apresentaram início de mudança de cor, os cachos tratados e não tratados foram ensacados com sacos de TNT (tecidos não tecidos).

A colheita dos cachos foi realizada quando o tratamento-testemunha atingiu $14^{\circ}$ Brix. No laboratório, os cachos foram submetidos a análises imediatas. As variáveis físicas analisadas foram: comprimento e largura dos cachos; diâmetro das bagas com paquímetro manual; número de bagas por cacho; massa dos cachos e bagas, em balança de precisão, e número de sementes por baga (amostra de 20 bagas). Para a análise química, foi utilizado o mosto de 20 bagas de cada parcela e avaliado o teor de sólidos solúveis totais (SST) com auxílio de refratômetro; acidez total titulável (ATT), pelo método de titulometria de neutralização com $\mathrm{NaOH} 0,1 \mathrm{~N}$, até atingir pH 8,1 com auxílio de phmetro digital e expressa em g de ácido tartárico por $100 \mathrm{~mL}$ de mosto (INSTITUTO ADOLFO LUTZ, 1985); pH por meio de phmetro digital e relação teor de sólidos solúveis totais/acidez total titulável (SST/ATT). A análise sensorial foi realizada nas uvas colhidas na segunda safra, que receberam aplicação de $\mathrm{GA}_{3} 100 \mathrm{mg} \mathrm{L}^{-1}$ e uvas da testemunha (sem aplicação de regulador vegetal). Foram realizadas duas sessões: a primeira foi um treinamento e a segunda foi a avaliação propriamente dita. Os julgadores receberam uma ficha de avaliação de Análise Descritiva Quantitativa (ADQ) (ABNT, 1993), com uma escala não estruturada de $10 \mathrm{~cm}$ (de ruim a ótimo). Os atributos avaliados foram: tamanho da baga, cor, aroma, textura, adstringência, acidez, doçura e sabor.

Os resultados foram submetidos à análise de variância e comparação de médias, pelo teste de Tukey, a 5\% de probabilidade, pelo programa estatístico SISVAR versão 5.3.

Pelo fato de os produtos químicos TDZ e BAP não possuírem registro para a cultura da videira, foi realizado como complementação ao estudo um teste residual destes produtos pelo TECPAR (Instituto de Tecnologia do Paraná) nos tratamentos que receberam os produtos.

\section{RESULTADOS E DISCUSSÃO}

A uva 'Bordô' demonstrou real possibilidade de cultivo para a produção de suco e vinho, uma vez que a testemunha (Tabela 1), ao contrário de informações preliminares de produtores da região, apresentou valores de SST e relação SST/ATT que se enquadram nos valores estabelecidos pela legislação, mínimo de $14^{\circ}$ Brix e relação entre 15 e 45 (BRASIL, 2004). Apesar deste fato, a aplicação de $\mathrm{GA}_{3}(100$ $\mathrm{mg} \mathrm{L}^{-1}$ ) propiciou maior teor de SST, e menor ATT, 
o que levou ao aumento da relação SST/ATT (Tabela 1) e, em consequência, melhorou o produto para a produção de vinho e suco de uva. Estes resultados discordam de Nachtigal et al. (2005) e Zoffoli et al.(2009), que encontraram diminuição do teor de SST com a aplicação de $\mathrm{GA}_{3}$. Nachtigal et al. (2005) efetuaram duas aplicações de 30; 60 e $90 \mathrm{mg} \mathrm{L}^{-1}$ de $\mathrm{GA}_{3}$ em uva 'BRS Clara' (Vitis vinifera), e Zoffoli et al.(2009) efetuaram uma aplicação de $40 \mathrm{mg} \mathrm{L}^{-1}$ de $\mathrm{GA}_{3}$ em uva 'Redglobe Santiago' (Vitis vinifera). Tais discordâncias podem estar relacionadas ao fato de que, na viticultura, as variedades podem responder de forma diferenciada à aplicação de reguladores vegetais.

Nas demais características físicas avaliadas, a aplicação de $\mathrm{GA}_{3}$ propiciou o melhor resultado nas duas safras, com aumento da massa média e aumento no diâmetro de bagas (Tabelas 2 e 3), concordando com a pesquisa de Gowda et al. (2006), em que, em razão do efeito dos reguladores vegetais, as bagas de uva expandem-se pelo aumento da atividade de divisão celular em fase inicial do desenvolvimento e pelo influxo de água e transporte de metabólicos na fase final do desenvolvimento dos frutos. Respostas similares foram encontradas em trabalhos que avaliaram o efeito de $20 \mathrm{mg} \mathrm{L}^{-1}$ de $\mathrm{GA}_{3}$ em uva 'Kyoho' (BYIN; KIM, 1995), 60 e $90 \mathrm{mg} \mathrm{L}^{-1}$ de $\mathrm{GA}_{3}$ em uva 'BRS Clara' (NACHTIGAL et al., 2005), e 30 $\mathrm{mg} \mathrm{L}^{-1}$ de $\mathrm{GA}_{3}$ em uva 'Itália' (RODRIGUES et al., 2011).

As aparentemente diferentes características físico-químicas das uvas produzidas nas duas também foram encontradas por Scarpare Filho et al. (2003), Cato et al.(2005) e Rodrigues et al. (2011). Estes autores justificam estas diferenças devido ao fato de que a ação dos reguladores vegetais está diretamente relacionada às condições climáticas e épocas de aplicação.

Os tratamentos com TDZ (10) e TDZ (5) + $\mathrm{GA}_{3}(100)$ ocasionaram aumento significativo da ATT, chegando perto do limite estipulado pela legis- lação de $0,90 \mathrm{~g}$ \%. Isto levou a uma diminuição significativa da relação SST/ATT em relação à testemunha (Tabela 1), piorando a qualidade para a produção de suco e vinho. Esses resultados discordam de Botelho et al. (2002), que observaram que a aplicação de TDZ (5 e $10 \mathrm{mg} \mathrm{L}^{-1}$ ) em uva 'Venus' não alterou a relação SST/ATT do mosto.

$\mathrm{O}$ TDZ e o BAP ainda não possuem registro para a cultura da videira no Brasil, não podendo ser avaliados sensorialmente, tampouco serem recomendados para o uso comercial, entretanto foram testados neste trabalho por haver uma perspectiva de que também iriam melhorar as características físico-químicas da uva 'Bordô'. Porém, quando comparados ao GA, não apresentaram superioridade. Quanto ao teste complementar para resíduo, foram encontrados para o TDZ na concentração de $5 \mathrm{mg} \mathrm{L}^{-1}$ resíduo de $11,1 \mu \mathrm{g}$ $\mathrm{kg}^{-1}$, TDZ na concentração de $10 \mathrm{mg} \mathrm{L}^{-1}$ resíduo de $9,9 \mu \mathrm{g} \mathrm{kg}^{-1}$, BAP na concentração de $5 \mathrm{mg} \mathrm{L}^{-1}$ resíduo de $4,2 \mu \mathrm{gkg}^{-1}$ e BAP na concentração de $10 \mathrm{mg} \mathrm{L}^{-1}$ resíduo de $7,2 \mu \mathrm{g} \mathrm{kg}^{-1}$. Não foram encontrados na legislação quais são os limites máximos de resíduo destes produtos em alimentos.

Considerando a possibilidade de que estas uvas possam ser destinadas ao consumo in natura, foi efetuada uma análise sensorial da testemunha, comparativamente com o $\mathrm{GA}_{3}$ o único produto cujo uso é registrado para a cultura da uva. Apesar de não haver diferença significativa entre aplicação de $\mathrm{GA}_{3}$ a $100 \mathrm{mg} \mathrm{L}^{-1}$ e a testemunha, os frutos que receberam aplicação de $\mathrm{GA}_{3}$ foram mais bem avaliados em todas as características (Figura 1). Isto se deve certamente ao efeito positivo do aumento da relação SST/ATT no sabor e do aumento do tamanho das bagas, concordando com Lulu et al.(2005), quando ele cita que, no mercado brasileiro de uvas de mesa, percebe-se uma exigência cada vez maior dos consumidores nacionais por uvas de melhor qualidade, não somente quanto ao aspecto físico, mas também ao sabor e aroma. 
TABELA 1 - Características químicas da uva cv. Bordô tratados com reguladores vegetais $\left(\mathrm{GA}_{3}=\right.$ ácido giberélico, TDZ = thidiazuron e BAP = 6-benzilaminopurina) em duas safras, 2009/2010 e 2010/2011. Bocaiuva do Sul-PR.

\begin{tabular}{|c|c|c|c|c|c|c|c|}
\hline \multirow{2}{*}{$\begin{array}{l}\text { Tratamento } \\
\left(\mathrm{mg} . \mathrm{L}^{1}\right)\end{array}$} & \multicolumn{2}{|c|}{ SST $\left({ }^{\circ} \mathrm{BRIX}\right)$} & \multicolumn{2}{|c|}{ ATT $\left(\mathrm{g} 100 \mathrm{~mL}^{-1}\right)$} & \multicolumn{2}{|c|}{ Relação SST/ATT } & \multirow{2}{*}{$\begin{array}{c}\mathrm{pH} \\
2010 / 2011\end{array}$} \\
\hline & $2009 / 2010$ & $2010 / 2011$ & $2009 / 2010$ & $2010 / 2011$ & $2010 / 2011$ & $2009 / 2010$ & \\
\hline Testemunha (0) & $16,30 \mathrm{ab}$ & $14,90 \mathrm{~d}$ & $0,64 \mathrm{c}$ & $0,68 \mathrm{a}$ & $21,42 \mathrm{~cd}$ & $3,53 \mathrm{abc}$ & $3,55 \mathrm{abc}$ \\
\hline TDZ ( 5 ) & $15,73 \mathrm{ab}$ & $15,15 \mathrm{~cd}$ & $0,68 \mathrm{bc}$ & $0,67 \mathrm{ab}$ & $22,91 \mathrm{~cd}$ & $3,47 \mathrm{bc}$ & $3,62 \mathrm{abc}$ \\
\hline TDZ (10) & $16,23 \mathrm{ab}$ & $16,15 \mathrm{bc}$ & $0,76 \mathrm{ab}$ & $0,56 \mathrm{bcd}$ & $28,79 \mathrm{ab}$ & $3,48 \mathrm{abc}$ & $3,53 \mathrm{abc}$ \\
\hline $\mathrm{TDZ}(5)+\mathrm{GA}_{3}(100)$ & $15,46 \mathrm{~b}$ & $17,20 \mathrm{ab}$ & $0,82 \mathrm{a}$ & $0,51 \mathrm{~d}$ & $33,15 \mathrm{a}$ & $3,53 \mathrm{abc}$ & $3,65 \mathrm{a}$ \\
\hline $\mathrm{TDZ}(10)+\mathrm{GA}_{3}(100)$ & $15,33 \mathrm{~b}$ & $14,95 \mathrm{~cd}$ & $0,66 \mathrm{bc}$ & $0,72 \mathrm{a}$ & $20,66 \mathrm{~d}$ & $3,56 \mathrm{abc}$ & $3,48 \mathrm{bc}$ \\
\hline $\mathrm{GA}_{3}(100)$ & $17,13 \mathrm{a}$ & $17,80 \mathrm{a}$ & $0,37 \mathrm{~d}$ & $0,55 \mathrm{~cd}$ & 32,56 a & $3,52 \mathrm{abc}$ & $3,60 \mathrm{abc}$ \\
\hline BAP (5) & $15,66 \mathrm{~b}$ & $17,05 \mathrm{ab}$ & $0,60 \mathrm{c}$ & $0,68 \mathrm{a}$ & $25,01 \mathrm{bcd}$ & $3,57 \mathrm{ab}$ & $3,46 \mathrm{c}$ \\
\hline BAP (10) & $16,40 \mathrm{ab}$ & $15,70 \mathrm{~cd}$ & $0,67 \mathrm{bc}$ & $0,72 \mathrm{a}$ & $22,11 \mathrm{~cd}$ & $3,46 \mathrm{c}$ & $3,47 \mathrm{bc}$ \\
\hline $\mathrm{BAP}(5)+\mathrm{GA}_{3}(100)$ & $16,53 \mathrm{ab}$ & $16,95 \mathrm{ab}$ & $0,65 \mathrm{c}$ & $0,55 \mathrm{~cd}$ & $31,02 \mathrm{a}$ & $3,59 \mathrm{a}$ & $3,63 \mathrm{ab}$ \\
\hline $\operatorname{BAP}(10)+\mathrm{GA}_{3}(100)$ & $15,93 \mathrm{ab}$ & $17,00 \mathrm{ab}$ & $0,68 \mathrm{bc}$ & $0,66 a b c$ & $25,92 \mathrm{bc}$ & $3,51 \mathrm{abc}$ & $3,52 \mathrm{abc}$ \\
\hline $\mathrm{CV} \%$ & 4,97 & 3,06 & 8,85 & 7,36 & 7,55 & 1,56 & 1,91 \\
\hline
\end{tabular}

Médias seguidas da mesma letra minúscula na coluna não diferem entre si, pelo teste de Tukey $(\mathrm{p}<0,05)$.

TABELA 2 - Características físicas da uva cv. Bordô tratadas com reguladores vegetais $\left(\mathrm{GA}_{3}=\right.$ ácido giberélico, TDZ = thidiazuron e BAP = 6-benzilaminopurina) em duas safras, 2009/2010 e 2010/2011. Bocaiuva do Sul-PR.

\begin{tabular}{|c|c|c|c|c|c|c|}
\hline \multirow{2}{*}{$\begin{array}{l}\text { Tratamento } \\
\left(\mathrm{mg} . \mathrm{L}^{1}\right)\end{array}$} & \multicolumn{2}{|c|}{$\begin{array}{l}\text { Massa média dos cachos } \\
(\mathrm{g})\end{array}$} & \multicolumn{2}{|c|}{$\begin{array}{c}\text { Massa média das bagas } \\
(\mathrm{g})\end{array}$} & \multicolumn{2}{|c|}{$\begin{array}{l}\text { Número médio de } \\
\text { bagas por cacho }\end{array}$} \\
\hline & $2009 / 2010$ & $2010 / 2011$ & $2009 / 2010$ & $2010 / 2011$ & $2009 / 2010$ & $2010 / 2011$ \\
\hline Testemunha (0) & $89,50 \mathrm{~b}$ & $119,52 \mathrm{ab}$ & $89,33 \mathrm{~b}$ & $115,37 \mathrm{ab}$ & 35,08 a & $52,25 \mathrm{a}$ \\
\hline TDZ ( 5 ) & $117,16 \mathrm{ab}$ & $103,35 \mathrm{ab}$ & $113,16 a b$ & $97,94 \mathrm{ab}$ & 44,75 a & $39,87 \mathrm{abc}$ \\
\hline TDZ (10) & $116,08 \mathrm{ab}$ & $89,72 \mathrm{ab}$ & $112,58 \mathrm{ab}$ & $85,10 \mathrm{ab}$ & $42,50 \mathrm{a}$ & $30,25 \mathrm{bc}$ \\
\hline $\mathrm{TDZ}(5)+\mathrm{GA}_{3}(100)$ & $136,83 \mathrm{a}$ & $92,49 \mathrm{ab}$ & $131,58 \mathrm{a}$ & $89,61 \mathrm{ab}$ & $46,25 \mathrm{a}$ & $31,00 \mathrm{bc}$ \\
\hline $\mathrm{TDZ}(10)+\mathrm{GA}_{3}(100)$ & 137,16 a & $118,72 \mathrm{ab}$ & 13 & 114 & 44 & $37,25 \mathrm{abc}$ \\
\hline $\mathrm{GA}_{3}(100)$ & $142,50 \mathrm{a}$ & $99,37 \mathrm{ab}$ & $137,33 \mathrm{a}$ & $96,06 \mathrm{ab}$ & 49,08 a & $36,87 \mathrm{abc}$ \\
\hline $\operatorname{BAP}(5)$ & $105,83 \mathrm{ab}$ & $128,54 \mathrm{ab}$ & $101,50 \mathrm{ab}$ & $124,44 \mathrm{ab}$ & 43,66 a & 54,87 a \\
\hline BAP (10) & $117,58 \mathrm{ab}$ & $86,10 \mathrm{ab}$ & $114,58 \mathrm{ab}$ & $82,33 \mathrm{ab}$ & $48,58 \mathrm{a}$ & $37,00 \mathrm{abc}$ \\
\hline $\mathrm{BAP}(5)+\mathrm{GA}_{3}(100)$ & $109,91 \mathrm{ab}$ & $72,38 \mathrm{~b}$ & $105,33 \mathrm{ab}$ & $68,13 \mathrm{~b}$ & $4075 \mathrm{a}$ & $26,12 \mathrm{c}$ \\
\hline $\mathrm{BAP}(10)+\mathrm{GA}_{3}(100)$ & $111,50 \mathrm{ab}$ & $136,40 \mathrm{a}$ & $107,50 \mathrm{ab}$ & $132,18 \mathrm{a}$ & $40,08 \mathrm{a}$ & $49,12 \mathrm{ab}$ \\
\hline $\mathrm{CV} \%$ & 16,57 & 24,34 & 16,94 & 24,54 & 18,73 & 21,08 \\
\hline
\end{tabular}

Médias seguidas da mesma letra minúscula na coluna não diferem entre si, pelo teste de Tukey $(\mathrm{p}<0,05)$. 
TABELA 3 - Características físicas da uva cv. Bordô tratada com reguladores vegetais $\left(\mathrm{GA}_{3}=\right.$ ácido giberélico, TDZ = thidiazuron e BAP = 6-benzilaminopurina) em duas safras, 2009/2010 e 2010/2011. Bocaiúva do Sul, PR.

\begin{tabular}{|c|c|c|c|c|c|c|c|c|}
\hline \multirow{2}{*}{$\begin{array}{l}\text { Tratamento } \\
\left(\mathrm{mg} \mathrm{L}^{-1}\right)\end{array}$} & \multicolumn{2}{|c|}{$\begin{array}{c}\text { Comprimento } \\
\text { médio cacho }(\mathrm{mm})\end{array}$} & \multicolumn{2}{|c|}{$\begin{array}{l}\text { Largura média } \\
\text { cacho }(\mathrm{mm})\end{array}$} & \multicolumn{2}{|c|}{$\begin{array}{l}\text { Diâmetro médio baga } \\
\qquad(\mathrm{mm})\end{array}$} & \multicolumn{2}{|c|}{$\begin{array}{l}\mathrm{N}^{\circ} \text { médio sementes } \\
\text { baga }\end{array}$} \\
\hline & $\begin{array}{l}2009 / \\
2010\end{array}$ & $\begin{array}{l}2010 / \\
2011\end{array}$ & $\begin{array}{l}2009 / \\
2010\end{array}$ & $\begin{array}{l}2010 / \\
2011\end{array}$ & $\begin{array}{l}2009 / \\
2010\end{array}$ & & $\begin{array}{l}2009 / \\
2010\end{array}$ & $\begin{array}{l}2010 / \\
2011\end{array}$ \\
\hline Teste & $10,00 \mathrm{a}$ & $12,81 \mathrm{a}$ & $7,00 \mathrm{a}$ & $7,45 \mathrm{a}$ & $15,40 \mathrm{bc}$ & 14 & $2,11 \mathrm{~d}$ & $2,73 \mathrm{~d}$ \\
\hline TDZ (5) & $10,18 \mathrm{a}$ & $11,06 \mathrm{a}$ & $7,24 \mathrm{a}$ & $7,93 \mathrm{a}$ & $16,08 \mathrm{ab}$ & $14,49 \mathrm{de}$ & $2,71 \mathrm{ab}$ & $2,58 \mathrm{de}$ \\
\hline TDZ $(10$ & $10,85 \mathrm{a}$ & $10,07 \mathrm{a}$ & $7,08 \mathrm{a}$ & $6,07 \mathrm{a}$ & $15,14 \mathrm{c}$ & 14,96 & $2,37 \mathrm{bcd}$ & $2,42 \mathrm{de}$ \\
\hline $\mathrm{TDZ}(5)+\mathrm{GA}_{3}(100)$ & $12,02 \mathrm{a}$ & $10,22 \mathrm{a}$ & $7,71 \mathrm{a}$ & $6,93 \mathrm{a}$ & 16,45 a & $15,24 \mathrm{ab}$ & $2,33 \mathrm{bcd}$ & $2,97 \mathrm{abc}$ \\
\hline $\mathrm{TDZ}(10)+\mathrm{GA}_{3}(100)$ & $10,65 \mathrm{a}$ & $11,40 \mathrm{a}$ & $7,60 \mathrm{a}$ & $7,43 \mathrm{a}$ & $16,58 \mathrm{a}$ & $15,96 \mathrm{abc}$ & $2,26 \mathrm{~cd}$ & $3,05 \mathrm{ab}$ \\
\hline $\mathrm{GA}_{3}(100)$ & $12,69 \mathrm{a}$ & $12,21 \mathrm{a}$ & $8,11 \mathrm{a}$ & $6,93 \mathrm{a}$ & $16,77 \mathrm{a}$ & $16,21 \mathrm{ab}$ & $2,38 \mathrm{bcd}$ & $2,97 \mathrm{abc}$ \\
\hline BAP (5) & $10,11 \mathrm{a}$ & $10,76 \mathrm{a}$ & 7,0 & $7,87 \mathrm{a}$ & 15,57 & $15,16 \mathrm{bcd}$ & $2,41 \mathrm{bcd}$ & $2,91 \mathrm{bc}$ \\
\hline BAP (10) & $10,39 \mathrm{a}$ & $10,37 \mathrm{a}$ & $7,55 \mathrm{a}$ & $6,85 \mathrm{a}$ & $16,06 \mathrm{ab}$ & 15,52 abcd & $2,89 \mathrm{a}$ & $2,58 \mathrm{de}$ \\
\hline $\mathrm{BAP}(5)+\mathrm{GA}_{3}(100)$ & $10,07 \mathrm{a}$ & $9,67 \mathrm{a}$ & $7,57 \mathrm{a}$ & $6,20 \mathrm{a}$ & $16,43 \mathrm{a}$ & $16,31 \mathrm{a}$ & $2,38 \mathrm{bcd}$ & $3,25 \mathrm{a}$ \\
\hline $\operatorname{BAP}(10)+\mathrm{GA}_{3}(100)$ & $10,82 \mathrm{a}$ & $12,21 \mathrm{a}$ & $6,60 \mathrm{a}$ & 7,96 a & $16,15 \mathrm{ab}$ & $16,00 \mathrm{abc}$ & $2,55 \mathrm{abc}$ & $3,2 \mathrm{ab}$ \\
\hline$C v 70$ & 13,52 & 12,20 & 14,49 & 12,90 & 2,51 & 2,92 & 8,25 & 4,38 \\
\hline
\end{tabular}

Médias seguidas da mesma letra minúscula na coluna não diferem entre si, pelo teste de Tukey $(\mathrm{p}<0,05)$.

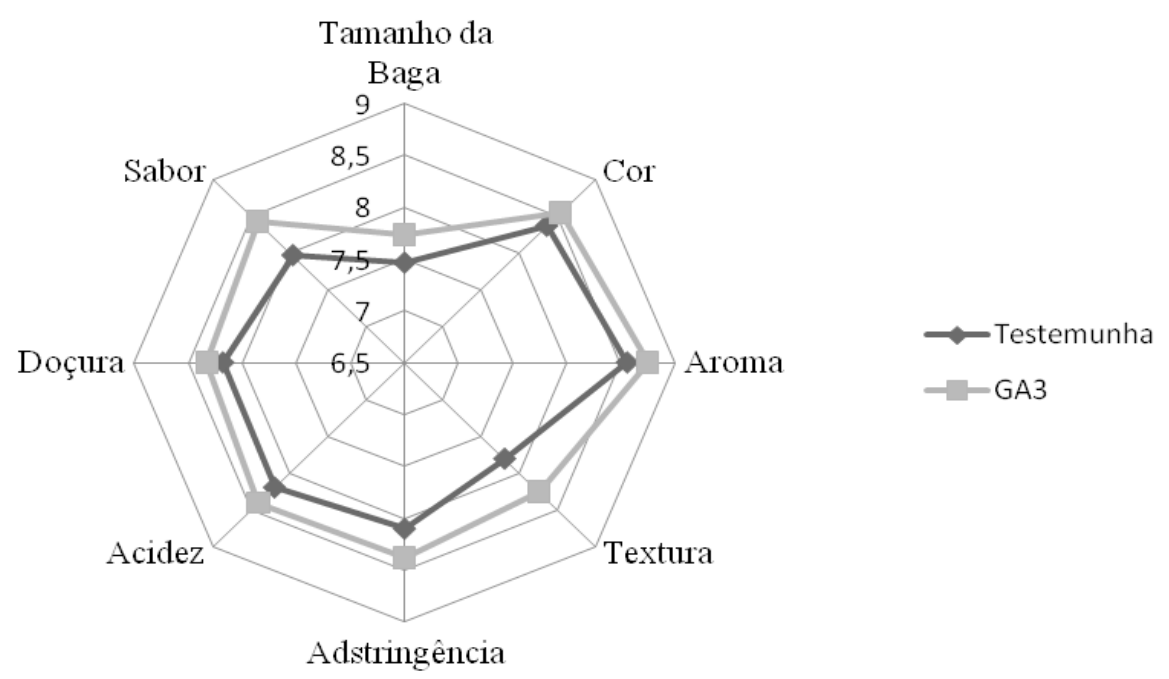

FIGURA 1 - Descritores sensoriais da uva "Bordô". 


\section{CONCLUSÃO}

A aplicação de $\mathrm{GA}_{3}$ a $100 \mathrm{mg} \mathrm{L}^{-1}$ propiciou melhoria da qualidade dos frutos de uva 'Bordô' para a produção de vinho e suco de uva, além de maior desenvolvimento dos frutos.

\section{REFERENCIAS}

ABNT - Associação Brasileira de Normas Técnicas. NBR 14140: alimentos e bebidas - análise sensorial - teste de análise descritiva quantitativa (ADQ). Rio de Janeiro, 1993.

BOTELHO, R.V.; PIRES, E.J.P.; TERRA, M.M. Qualidade da uva de mesa'Vênus' tratada com Thidiazuron. Revista Ceres, Lavras, v. 49, n. 286, p. 629-639, 2002.

BOTELHO, R. V.; PIRES, E. J. P.; TERRA, M. $M$. Efeitos de reguladores vegetais na qualidade de uvas 'Niagara Rosada' na região noroeste do Estado de São Paulo. Revista Brasileira de Fruticultura, Jaboticabal, v. 26, n. 1, p. 74-77. 2004.

BRASIL. Ministério da Agricultura. Secretaria Nacional de Defesa Agropecuária. Complementação de padrões de identidade e qualidade do vinho e dos derivados da uva e do vinho. Brasília: MAPA, 2004. $21 \mathrm{p}$.

BYUN, J.K.; KIM, J.S. Effects of GA3, thidiazuron and ABA on fruit set and quality of 'Kyoho' grapes. Journal of the Korean Society for Horticultural Science, Kyongsan, v.36, n.2, p.231-239, 1995.

CAMARGO, U.A.; MAIA, J. D. G.; NACHTIGAL, J. BRS VIOLETA. Nova cultivar de uva para suco e vinho de mesa. Bento Gonçalves: Embrapa Uva e Vinho, 2005. 8 p. (Comunicado Técnico, 63).

CATO, S.C.; TERRA, M.M.; BOTELHO, R.V.; TECCHIO, M.A.; PIRES, E.J.P.; CARVALHO, C.R.L.; PIEDADE, S.M.S. Características morfológicas dos cachos e bagas de uva 'Niagara Rosada' (Vitis labrusca L.) tratadas com o ácido giberélico e anelamento. Acta Scientiarum, Agronomy, Maringá, v.27, n.1, p.177-181, 2005.

CASANOVA, L.; CASANOVA, R.; MORET, A.; AGUSTÍ, M. The application of gibberellic acid increases berry size of 'Emperatriz' seedless grape. Spanish Journal of Agricultural Research, Madrid, v.7, n.4, p.919-927, 2009.
CHOUDHURY, M. M. (Ed.). Uva de mesa: pós-colheita. Brasília: Embrapa Informação Tecnológica; Petrolina-PE: Embrapa Semiárido, 2001. 55 p. (Frutas do Brasil, 12).

GOWDA, V.N.; SHYAMALAMMA, S ; KANNOLLI,R.B. Influence of GA3 on growth and development of 'Thompson Seedless' grapes (Vitis vinifera L.).Acta Horticulturae, The Hague, n.727, p.239-242, 2006.

INSTITUTO ADOLFO LUTZ. Normas analíticas do Instituto Adolfo Lutz: métodos químicos e físicos para análise dos alimentos. 3. ed. São Paulo, 1985. v.1, $371 \mathrm{p}$.

LULU, J.; CASTRO, J.V.; PEDRO JÚNIOR, M. J. Efeito do microclima na qualidade da uva de mesa 'Romana' (A1105) cultivada sob cobertura plástica. Revista Brasileira de Fruticultura, Jaboticabal, v. 27, n. 3, p. 422-425, 2005.

NACHTIGAL, J.C.; CAMARGO, U.A.; MAIA, J.D.G. Efeito de reguladores de crescimento em uva apirênica, cv. BRS Clara. Revista Brasileira de Fruticultura, Jaboticabal, v. 27, n. 2, p. 304-307, 2005.

REGINA, M.A.; CARMO, E.L.; FONSECA, A.R.; PURGATTO, E.; SHIGA, T.M.; LAJOLO, F.M.; RIBEIRO, A.P.; MOTA, R.V. Influência da altitude na qualidade das uvas 'Chardonnay' e 'Pinot Noir' em Minas Gerais. Revista Brasileira de Fruticultura, Jaboticabal, v. 32, n. 1, p. 143-150, 2010.

RIZZON, L. A.; MIELE, A.; MENEGUZZO, J. Avaliação da uva cv. Bordô para a elaboração de vinho tinto. Ciência e Tecnologia de Alimentos, Campinas, v. 20, n. 1, p. 115-121, 2000.

RIZZON, L. A.; MENEGUZZO, J.; MANFROI, L. Processamento de uva, vinho tinto, graspa e vinagre. Brasília: Embrapa Informação Tecnológica, 2004.

RIZZON, L. A.; LINK, M. Composição do suco de uva caseiro de diferentes cultivares. Ciência Rural, Santa Maria, v. 36, n. 2, p. 689-692, 2006.

RIZZON, L.A; MIELE, A. Efeito da safra vitícola na composição da uva, do mosto e do vinho Isabel na Serra Gaúcha, Brasil. Ciência Rural, Santa Maria, v. 36, n. 3, p. 959-964, 2006. 
RODRIGUES, A.; ARAÚJO, J.P.C.; GIRARDI, E.A.; SCARPARE, F.V.; SCARPARE FILHO, J.L. Aplicação de $\mathrm{AG}_{3}$ e CPPU na qualidade da uva 'Itália' em Porto Feliz-SP. Revista Brasileira de Fruticultura, Jaboticabal, v. 33, n. 1, p. 001-007, 2011.

SCARPARE FILHO, J. A. ; RIBEIRO, V. G. Crescimento de bagas de cultivares de uvas apirênicas tratadas com CPPU e GA3. Ciência e Agrotecnologia, Lavras, v. 27, n. 6, p. 1253-1259, 2003.

TECCHIO, M. A.; LEONEL, S.; CAMILIS, E. C.; MOREIRA, G. C.; PIRES, E. J. P.; RODRIGUES, J. D. Uso de bioestimulante na videira Niagara Rosada. Ciência e Agrotecnologia, Lavras, v. 30, n. 6, p.1236-1240, 2006.
VIEIRA, C.R.Y.I.; PIRES, E.J.P.; TERRA, M.M.; TECCHIO, M.A.; BOTELHO, R.V. Efeitos do ácido giberélico e do thidiazuron sobre as características dos frutos e do mosto da uva 'Niagara rosada'. Revista Brasileira de Fruticultura, Jaboticabal, v. 30, n. 1, p. 012-019, 2008.

ZOFOLLI, J.P.; LATORRE, B.A.; NARANJO, P. Preharvest applications of growth regulators and their effect on postharvest quality of table grapes during cold storage. Postharvest Biology and Technology, Amsterdam, v.52, n. 2, p. 183-192, 2009. 